OPEN ACCESS

Edited by:

Bilal Çiğ,

Ahi Evran University Medicine Faculty Department of Physiology, Turkey

Reviewed by: Alexei Verkhratsky, The University of Manchester, United Kingdom

Ikuko Miyazaki,

Okayama University, Japan Asuman Celikbilek,

Ahi Evran University, Turkey

*Correspondence: Rahul Srinivasan rahul@tamu.edu

Specialty section: This article was submitted to Integrative Physiology, a section of the journal Frontiers in Physiology

Received: 09 November 2021 Accepted: 09 December 2021

Published: 11 January 2022

Citation:

Bancroft EA and Srinivasan $R$ (2022) Emerging Roles for Aberrant Astrocytic Calcium Signals in Parkinson's Disease.

Front. Physiol. 12:812212. doi: 10.3389/fphys.2021.812212

\title{
Emerging Roles for Aberrant Astrocytic Calcium Signals in Parkinson's Disease
}

\section{Eric A. Bancroft ${ }^{1}$ and Rahul Srinivasan ${ }^{1,2 *}$ \\ 1 Department of Neuroscience and Experimental Therapeutics, Texas A\&M University College of Medicine, Bryan, TX, United States, ${ }^{2}$ Texas A\&M Institute for Neuroscience (TAMIN), College Station, TX, United States}

Astrocytes display a plethora of spontaneous $\mathrm{Ca}^{2+}$ signals that modulate vital functions of the central nervous system (CNS). This suggests that astrocytic $\mathrm{Ca}^{2+}$ signals also contribute to pathological processes in the CNS. In this context, the molecular mechanisms by which aberrant astrocytic $\mathrm{Ca}^{2+}$ signals trigger dopaminergic neuron loss during Parkinson's disease (PD) are only beginning to emerge. Here, we provide an evidence-based perspective on potential mechanisms by which aberrant astrocytic $\mathrm{Ca}^{2+}$ signals can trigger dysfunction in three distinct compartments of the brain, viz., neurons, microglia, and the blood brain barrier, thereby leading to PD. We envision that the coming decades will unravel novel mechanisms by which aberrant astrocytic $\mathrm{Ca}^{2+}$ signals contribute to PD and other neurodegenerative processes in the CNS.

Keywords: Parkinson's disease, astrocytes, calcium, mitochondria, neurodegenenerative diseases

\section{INTRODUCTION}

Astrocytes are ubiquitous cells of the central nervous system (CNS) that outnumber neurons in many brain regions (von Bartheld et al., 2016). These cells are important players in governing neuronal function via mechanisms such as synaptic pruning, neurotransmitter clearance, and extracellular $\mathrm{K}^{+}$buffering (Verkhratsky and Nedergaard, 2018). The critical role played by astrocytes in CNS function makes it vitally important to understand molecular mechanisms underlying bidirectional communication between astrocytes and neurons.

\section{Astrocytic $\mathrm{Ca}^{2+}$ Signals Are Important for Normal Central Nervous System Function}

Unlike neurons, astrocytes are not electrically excitable, which has necessitated inquiry into the molecular machinery utilized by astrocytes to exert their functional effects on neurons and neural circuits. In this regard, studies utilizing genetically encoded calcium indicators (GECIs) such as GCaMPs have shown that astrocytes possess a plethora of spontaneous $\mathrm{Ca}^{2+}$ signals in situ and in vivo. Astrocytic $\mathrm{Ca}^{2+}$ signals respond to a variety of pharmacological and behavioral stimuli (Semyanov et al., 2020), and are observed in intracellular compartments such as the soma, thick proximal branches, and fine astrocytic processes (Srinivasan et al., 2015). In addition, astrocytic $\mathrm{Ca}^{2+}$ signals occur via $\mathrm{Ca}^{2+}$ release from distinct subcellular organelles such as the endoplasmic reticulum (ER; Okubo et al., 2019) and mitochondria (Huntington and Srinivasan, 2021), as well as extracellular $\mathrm{Ca}^{2+}$ sources (Srinivasan et al., 2015). At a subcellular level, the mechanisms governing astrocytic $\mathrm{Ca}^{2+}$ signals in the soma are distinct from those in peripheral processes 
(Verkhratsky et al., 2020). For example, $\mathrm{Ca}^{2+}$ signals in the soma and primary astrocytic processes occur due to metabotropic receptor activity, $\mathrm{InsP}_{3}$-mediated release of $\mathrm{Ca}^{2+}$ from the $\mathrm{ER}$ and store-operated $\mathrm{Ca}^{2+}$ entry. On the other hand, $\mathrm{Ca}^{2+}$ signals in fine astrocytic processes depend on mitochondrial $\mathrm{Ca}^{2+}$ fluxes, ionotropic channels such as transient receptor potential (TRP) and reversal of the $\mathrm{Na}^{+} / \mathrm{Ca}^{2+}$ exchanger, NCX. The presence of distinct compartments, mechanisms and sources for astrocytic $\mathrm{Ca}^{2+}$ signals strongly suggests that these signals modulate a diverse array of signaling pathways not only in the astrocytes themselves, but also in the neural circuits within which they reside.

Critical roles for spontaneous astrocytic $\mathrm{Ca}^{2+}$ signals in CNS function are bolstered by studies showing that these signals regulate the probability of neurotransmitter release (Covelo and Araque, 2018), long-term potentiation (Shigetomi et al., 2013; Arizono et al., 2020), maintenance of blood brain barrier (BBB) integrity (Heithoff et al., 2021), neurotransmitter clearance (Shigetomi et al., 2011; Haustein et al., 2014), and the synchronization and integration of neural activity (Sasaki et al., 2014; Pirttimaki et al., 2017; Deemyad et al., 2018). Since these processes are vitally important for normal CNS function, it is likely that a disruption in spontaneous astrocytic $\mathrm{Ca}^{2+}$ signaling is potentially pathological. In this regard, a particularly interesting question is how aberrant astrocytic $\mathrm{Ca}^{2+}$ signals could contribute to neurodegeneration.

\section{Aberrant Astrocytic $\mathrm{Ca}^{2+}$ Signaling and Parkinson's Disease}

Given their central role in brain function, it is not surprising that pathological alterations in astrocytes can accelerate the evolution of a variety of neurological diseases (Verkhratsky et al., 2017). Indeed, neurodegenerative diseases such as amyotrophic lateral sclerosis (ALS), and Alzheimer's disease are characterized by distinct pathological changes in astrocytes. Examples of this include impaired glutamate uptake and death of motor neurons in ALS (Rossi et al., 2008; Valori et al., 2014) or reduced astrocyte coverage in Alzheimer's disease, which results in synaptic deficiency and early cognitive dysfunction (Verkhratsky et al., 2016). Additionally, a recent study has shown that astrocytes derived from induced pluripotent stem cell (iPS) of Parkinson's disease (PD) patients with a leucine rich repeat kinase 2 (LRRK2) mutation display fragmented mitochondrial morphology, atrophic cellular morphology, altered $\mathrm{Ca}^{2+}$ signaling and metabolic impairment (RamosGonzalez et al., 2021). Together, these examples provide strong evidence for a central role of astrogliopathology in the evolution of neurodegenerative diseases.

Among the many known neurodegenerative disorders, $\mathrm{PD}$ is the second most common neurodegenerative disorder with no known cure (Poewe et al., 2017). PD is characterized by a progressive loss of substantia nigra pars compacta (SNc) dopaminergic (DA) neurons, and the onset of motor symptoms that include bradykinesia, resting tremors, postural instability, and muscle rigidity. Despite being labeled as a movement disorder, numerous non-motor symptoms are also observed during PD. These include sleep disturbances, constipation, anxiety, depression, and cognitive dysfunction (Poewe et al., 2017; Schapira et al., 2017). The complex clinical presentation of PD suggests a convergence of multiple mechanisms and cell types driving neurodegeneration. Most PD research, however, has focused on understanding pathological mechanisms that occur within the neurons themselves, without accounting for the role of astrocyte interactions with neurons, and other CNS cells during neurodegeneration. Consequently, neurocentric strategies have failed to result in the development of effective neuroprotective treatments for PD. In this context, we point to a central role for astrocytes, and more specifically, aberrant astrocytic $\mathrm{Ca}^{2+}$ signaling as an important contributing factor during the pathogenesis of PD.

Given the rapidly emerging importance of astrocytes in PD (Booth et al., 2017), as well as an urgent and unmet need to develop effective neuroprotective treatments, this review presents a perspective on potential mechanisms by which aberrant astrocytic $\mathrm{Ca}^{2+}$ signals can trigger, and possibly sustain neurodegeneration during the development of PD. We amalgamate recent independent reports to provide an evidencebased rationale for the role of aberrant astrocytic $\mathrm{Ca}^{2+}$ signals in pathologically altering three distinct elements of the CNS during $\mathrm{PD}$, viz. neurons, microglia, and the BBB (Figure 1).

\section{ABERRANT ASTROCYTIC $\mathrm{Ca}^{2+}$ SIGNALS CAN CAUSE DYSFUNCTION IN DOPAMINERGIC NEURONS}

Protoplasmic astrocytes possess a bushy morphology with primary branches that give rise to very fine secondary branches, branchlets and leaflets (Moye et al., 2019; Zhou et al., 2019). Fine processes from each astrocyte, can contact upwards of 150,000 synapses in rodents and over a million synapses in humans (Bushong et al., 2002; Oberheim et al., 2009; Semyanov and Verkhratsky, 2021). Based on the morphological relationship of astrocytic processes with neuronal synapses, spontaneous $\mathrm{Ca}^{2+}$ signals in astrocytic processes are optimally positioned to modulate neuronal function. In addition, the intimate morphological as well as functional relationship between astrocytes and neurons suggests that abnormal changes in $\mathrm{Ca}^{2+}$ signals within astrocytic processes can alter neuronal function and initiate neurodegeneration. In the sections below, we gather evidence from recent independent studies to illustrate exemplar mechanisms by which abnormal changes in astrocytic $\mathrm{Ca}^{2+}$ signals can trigger, and even sustain the degeneration of SNc DA neurons.

\section{Excitatory Amino Acid Transporter 2}

Excess extracellular glutamate is a major mechanism for neurodegeneration (Ambrosi et al., 2014; Lewerenz and Maher, 2015). This can occur via mechanisms such as glutamatemediated excitotoxicity (Lewerenz and Maher, 2015), oxidative glutamate toxicity (Schubert and Piasecki, 2001; Wang et al., 2020), and immunoexcitotoxicity (Blaylock, 2017). Astrocytes 


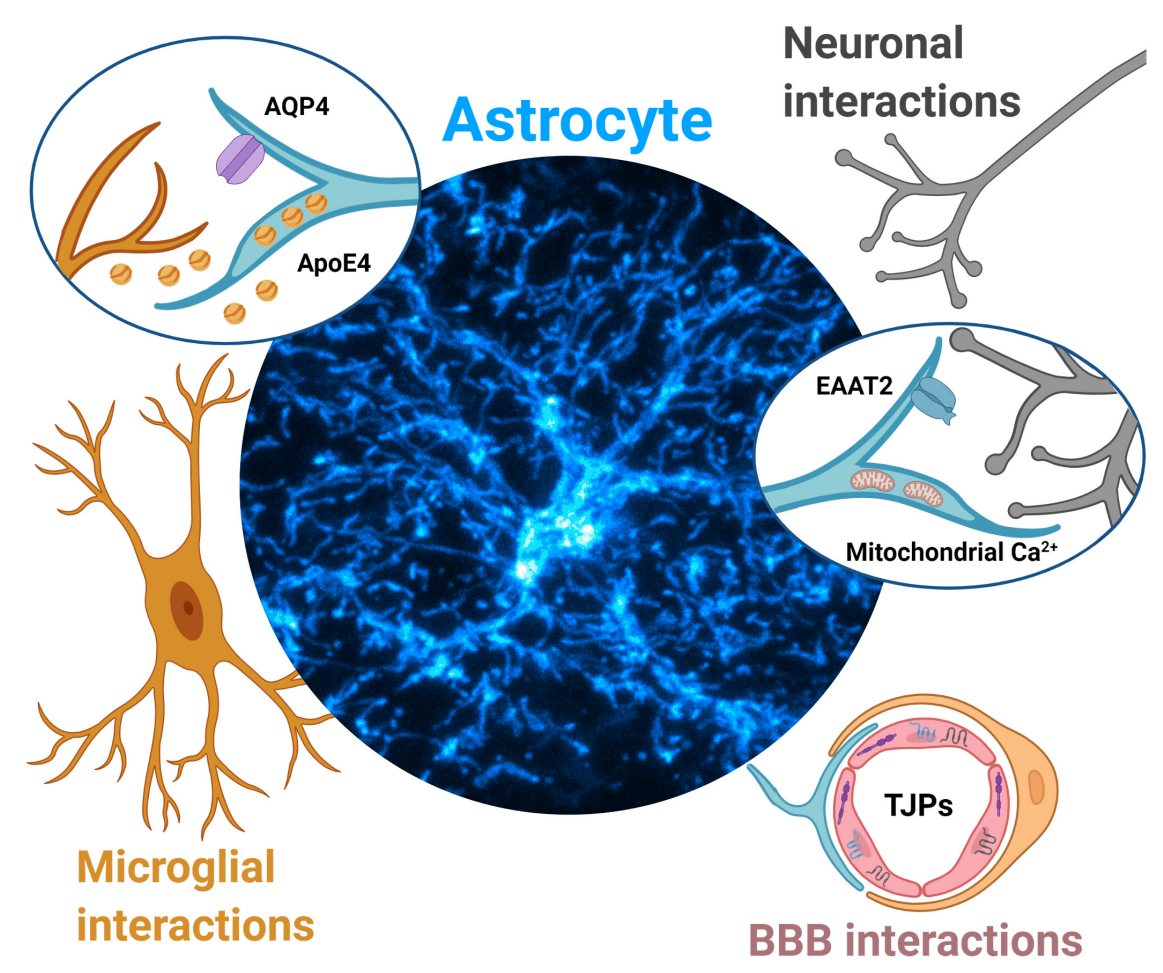

FIGURE 1 | Aberrant astrocytic $\mathrm{Ca}^{2+}$ signals contribute to Parkinson's disease pathology via multiple mechanisms. Neuronal interactions. Dopamine surges during early PD may dysregulate astrocytic $\mathrm{Ca}^{2+}$ signals and lead to EAAT2 internalization, leading to reduced glutamate clearance and initiation of excitotoxic cell death for dopaminergic midbrain neurons. Disruptions in astrocytic mitochondria $\mathrm{Ca}^{2+}$ signals (triggered from protein aggregates such as $\alpha$-synuclein) lead to reduced ATP production, alteration of mitochondria-ER tethering, likely contributing to dopaminergic neurodegeneration. Microglial interactions. Aberrant astrocytic Ca ${ }^{2+}$ signals drive mislocalization of AQP4 channels in astrocytes. AQP4 deficiency in astrocytes is associated with increases in microglial activity and further secretion of inflammatory cytokines, ultimately contributing to dopaminergic neurodegeneration. Aberrant astrocytic $\mathrm{Ca}^{2+}$ signals may drive increased secretion of ApoE4 which leads to microglial reactivity, increased $\alpha$-synuclein pathology and eventually dopaminergic neurodegeneration. Blood brain barrier (BBB) interactions. Aberrant Ca ${ }^{2+}$ signals in astrocyte endfeet may result in altered secretion of neurotrophic factors such as GDNF, leading to dysregulation of tight junction proteins (TJPs), compromised BBB integrity, and further contribute to dopaminergic neurodegeneration.

play a major role in neurotransmitter clearance (Eulenburg and Gomeza, 2010) and specifically, glutamate clearance via astrocytic glutamate transporters such as excitatory amino acid transporter 2 (EAAT2) (Lehre and Danbolt, 1998). Therefore, any reduction in astrocytic EAAT2 expression would result in abnormal levels of extracellular glutamate and neurodegeneration. Indeed, reductions in astrocytic EAAT2 expression are observed in multiple neurodegenerative diseases such as amyotrophic lateral sclerosis, Alzheimer's disease and Huntington's disease (Bruijn et al., 1997; Tong et al., 2014; Sharma et al., 2019). With regard to PD, two recent pieces of evidence are particularly relevant: (i) The targeted knockdown of EAAT2 in astrocytes causes degeneration of SNc DA neurons in a mouse model of PD (Zhang et al., 2020) and (ii) Exposure of rodents to the PD toxin 6hydroxydopamine (6-OHDA) causes a downregulation of EAAT2 (Chotibut et al., 2017).

A recent study shows that EAAT2 internalization from the surface of astrocytes increases in a $\mathrm{Ca}^{2+}$-dependent manner (Ibanez et al., 2019). Specifically, $\mathrm{Ca}^{2+}$ influx via the NCX sodium/calcium exchanger in response to increases in extracellular glutamate results in EAAT2 internalization. In a broader sense, one could infer that increased $\mathrm{Ca}^{2+}$ influx within astrocytes due to abnormal increases in extracellular neurotransmitters could result in EAAT2 internalization. We rationalize that a surge in striatal dopamine levels during early $\mathrm{PD}$, as seen in the Thy1- $\alpha$-synuclein mouse model of PD (Lam et al., 2011) can cause a downregulation of EAAT2 in striatal astrocytes. A recent study by Adermark et al. (2021) showed that pre-treatment of striatal brain slices with the D2 dopamine receptor agonist, sulpiride prevented synaptic depression induced by the EAAT2 blocker, TFB-TBOA. These data suggest a rapid downregulation of EAAT2 function in striatal astrocytes due to an abnormal activation of striatal D2 receptors. In addition, studies have shown that synaptically released dopamine increases $\mathrm{Ca}^{2+}$ events in striatal astrocytes (Corkrum et al., 2020), and the activation of D2 receptors in ventral midbrain astrocytes causes a downregulation of EAAT2 expression (Xin et al., 2019).

When taken together, these studies point to aberrant dopamine-mediated $\mathrm{Ca}^{2+}$ signals in astrocytic processes as a potential mechanism for EAAT2 downregulation in astrocytes leading to excess extracellular glutamate and consequently, neurodegeneration. 


\section{Astrocytic Mitochondria}

Recent studies have shown that astrocytic processes contain mitochondria (Derouiche et al., 2015; Agarwal et al., 2017; Huntington and Srinivasan, 2021) and that mitochondria in fine astrocytic processes are closely associated with $\mathrm{Ca}^{2+}$ signals in their vicinity (Agarwal et al., 2017). Interestingly, the $\mathrm{Ca}^{2+}$ signals associated with astrocytic mitochondria are abnormally increased in a mouse model of amyotrophic lateral sclerosis expressing a mutant form of superoxide dismutase (SOD G93A) (Agarwal et al., 2017), suggesting a role for abnormal mitochondrial $\mathrm{Ca}^{2+}$ signaling in fine astrocytic processes during neurodegeneration. In addition, we have shown that mitochondria in astrocytic processes display spontaneous $\mathrm{Ca}^{2+}$ influx with dual responses to neurotransmitter agonists, a dependency on $\mathrm{ER} \mathrm{Ca}^{2+}$, and the absence of functional mitochondrial uniporters (MCU; Huntington and Srinivasan, 2021), suggesting that astrocytic mitochondria possess unique functional properties that optimally cater to the extensive energy needs of DA neurons. A significant proportion ( 25\%) of energy demands in the CNS are met by astrocytes (van Hall et al., 2009), and spontaneous astrocytic mitochondrial $\mathrm{Ca}^{2+}$ signals derived from the endoplasmic reticulum (ER) activate mitochondrial dehydrogenases in order to generate the co-factors required for oxidative phosphorylation and ATP generation (Cardenas et al., 2010). Disruption of $\mathrm{Ca}^{2+}$ signals in astrocytic mitochondria could therefore be a harbinger for neurodegeneration.

Given the important role of $\alpha$-synuclein ( $\alpha$-syn) in the pathogenesis of $\mathrm{PD}$, it is pertinent to discuss aberrant $\mathrm{Ca}^{2+}$ signals in mitochondria within astrocytic processes as it relates to $\alpha$-syn pathology. Recent studies have shown that astrocytes readily take up extracellular $\alpha$-syn aggregates, and that $\alpha$-syn can damage astrocytic mitochondria (Lindstrom et al., 2017), as well as alter molecular tethering between the mitochondria and $\mathrm{ER}$, resulting in disrupted $\mathrm{Ca}^{2+}$ homeostasis (Paillusson et al., 2017). Together, these reports suggest that pathological forms of $\alpha$-syn can bind to astrocytic mitochondria, which could alter mitochondrial $\mathrm{Ca}^{2+}$ signaling in astrocytes, thereby causing a significant reduction in ATP generation and DA neuron loss. It is also important to note that astrocytes are coupled via gap junctions (Fujii et al., 2017). Therefore, pathological changes in mitochondrial $\mathrm{Ca}^{2+}$ signals in just a few astrocytes can affect larger populations of astrocytes within neural structures, thus magnifying the effects of aberrant astrocytic mitochondrial $\mathrm{Ca}^{2+}$ signaling on brain function and neurodegeneration.

\section{ABERRANT ASTROCYTIC $\mathrm{Ca}^{2+}$ SIGNALS CAN ACTIVATE MICROGLIA}

Microglia are classically viewed as the immune surveillance cells of the brain, with functions that include phagocytosis and synaptic pruning (Li and Barres, 2018; Bohlen et al., 2019; Bartels et al., 2020). In the context of DA neuron loss in PD, microglial reactivity is a strong indicator of neuroinflammation and ongoing neuropathology. There is evidence for microglial activation in clinical PD (McGeer et al., 1988; Gerhard et al., 2006; Bartels et al., 2010; Stokholm et al., 2017), as well as in rodent
(Czlonkowska et al., 1996; Wu et al., 2002; Sanchez-Guajardo et al., 2010; Hoenen et al., 2016) and non-human primate models of parkinsonism (McGeer et al., 2003; Barcia et al., 2004; Kanaan et al., 2008; Barkholt et al., 2012). There are potential mechanisms by which aberrant $\mathrm{Ca}^{2+}$ signals in astrocytes could play a role in initiating microglial activation during PD. In the sections below, we present two potential scenarios in which aberrant changes in spontaneous astrocytic $\mathrm{Ca}^{2+}$ signals could lead to abnormal crosstalk between astrocytes and microglia, thus accelerating neuronal loss in PD.

\section{Aquaporin 4}

Aquaporin 4 (AQP4) is a tetrameric water channel, abundantly expressed in astrocytes (Hubbard et al., 2015; Tham et al., 2016). Emerging evidence suggests a role for AQP4 dysfunction in PD. Studies supporting this idea include findings that: (i) Humans with Lewy body pathology in the neocortex demonstrate a negative correlation between AQP4 expressing astrocytes and $\alpha$-synuclein aggregates, such that astrocytes with AQP4 expression do not appear in areas with abnormal $\alpha$-synuclein expression (Hoshi et al., 2017), (ii) Exposure of AQP4 knockout (KO) mice to the PD toxin, MPTP causes an increase in the susceptibility of SNc DA neurons to degeneration (Fan et al., 2008), (iii) AQP4 KO mice show diminished differences between ventral tegmental area (VTA) and SNc DA neurons in their susceptibility to MPTP-induced neurodegeneration (Zhang et al., 2016), and (iv) AQP4 knockout mice display significant increases in microglial reactivity following exposure to MPTP when compared to wildtype littermates. In this case, the study also shows that the increase in microglial reactivity occurs due to secretion of neuroinflammatory molecules such as interleukin $1 \beta$ (IL1 $\beta)$ and tumor necrosis factor $\alpha$ (TNF $\alpha$; Sun et al., 2016). When taken together, these studies converge on the idea that a functional deficiency of AQP4 in astrocytes can result in microglial activation with a consequent increase in the secretion of neuroinflammatory molecules by activated microglia, in turn resulting in the loss of DA neurons.

As is the case for any channel, the ability of AQP4 to allow passage of water molecules through its pore requires precise localization at the plasma membrane. In this regard, studies show that AQP4 depends on $\mathrm{Ca}^{2+}$ for localization to the plasma membrane (Salman et al., 2017; Kitchen et al., 2020), and that rapid translocation of AQP4 to the plasma membrane depends on $\mathrm{Ca}^{2+}$ signals. Furthermore, a recent study has utilized STORM-based superresolution microscopy to show that AQP4 channels cluster in very specific patterns at astrocytic endfeet (Smith and Verkman, 2015). Thus, there exists an intricate relationship between $\mathrm{Ca}^{2+}$ signaling and the normal functional localization of AQP4 in astrocytes. Based on these data, one can infer that pathological changes in spontaneous astrocytic $\mathrm{Ca}^{2+}$ signals will result in the mislocalization and functional deficit of astrocytic AQP4, leading to microglial activation and neuroinflammation in the brain.

\section{Apolipoprotein E}

An allelic variant of the apolipoprotein E (ApoE) gene, ApoE4 significantly increases the risk for Alzheimer's disease (AD; 
Lambert et al., 2013; Liu et al., 2013; Yamazaki et al., 2019). In the case of PD, a recent study created ApoE locus-targeted ApoE4 replacement mice, and utilized these mice to show that ApoE4 increases $\alpha$-synuclein pathology, worsens behavioral deficits, and accelerates astrogliosis (Zhao et al., 2020). This study also showed that ApoE4 increases $\alpha$-synuclein pathology in $\mathrm{PD}$ patients.

In the CNS, astrocytes are a major reservoir for ApoE (Sun et al., 1998; Xu et al., 2006), and ApoE4 secretion occurs in a $\mathrm{Ca}^{2+}$-dependent manner (Kockx et al., 2007). Thus, any pathological alteration in the kinetics of astrocytic $\mathrm{Ca}^{2+}$ signals can alter the secretion of ApoE4 from astrocytes. Based on this rationale, increases in spontaneous astrocyte $\mathrm{Ca}^{2+}$ signal amplitudes as seen in reactive astrocytes (Shigetomi et al., 2019) could increase ApoE4 secretion by astrocytes, leading to microglial activation (Maezawa et al., 2006; Vitek et al., 2009), increased $\alpha$-synuclein uptake by microglia (Choi et al., 2020), the formation of toxic $\alpha$-synuclein aggregates (Davis et al., 2020) and neurodegeneration. Although we do not yet know what may initiate aberrant $\mathrm{Ca}^{2+}$ signaling in astrocytes, abnormal $\alpha$ synuclein uptake by astrocytes could disrupt $\mathrm{Ca}^{2+}$ homeostasis, and is therefore a likely candidate for triggering aberrant $\mathrm{Ca}^{2+}$ signals in SNc astrocytes during PD.

\section{ABERRANT ASTROCYTIC ENDFOOT $\mathrm{Ca}^{2+}$ SIGNALS AND BLOOD BRAIN BARRIER INTEGRITY}

The $\mathrm{BBB}$ is an important protective barrier that allows selective passage of molecules into the brain parenchyma. Abnormal increases in $\mathrm{BBB}$ permeability can allow the passage of environmental toxins into the midbrain, thereby accelerating DA neuron loss. This view is supported by the epidemiological finding that pesticide exposure is associated with an increased incidence of PD in farmers (Freire and Koifman, 2012). In this context, a histological study of striatal brain sections from PD patients has shown abnormal extravasation of erythrocytes, as well as an increase in extravascular serum proteins such as fibrin and hemoglobin into striatal parenchyma, suggesting a loss of BBB integrity during PD (Gray and Woulfe, 2015). Another recent study used dynamic contrast enhanced magnetic resonance imaging in $49 \mathrm{PD}$ patients to show significantly higher $\mathrm{BBB}$ leakage in posterior white matter regions of $\mathrm{PD}$ patients compared to healthy controls (Al-Bachari et al., 2020). These studies suggest that a loss of BBB integrity is likely involved in the pathogenesis of clinical PD.

The emerging evidence for a compromised $\mathrm{BBB}$ in $\mathrm{PD}$ patients motivates inquiry into whether or not astrocytes contribute to the maintenance of $\mathrm{BBB}$ integrity. In this regard, a recent study has utilized GLAST Cre/ERT2 mice driving the expression of the diphtheria toxin in astrocytes to ablate astrocytes in sparse regions of blood vessels. This study showed an extravasation of cadaverine from blood vessels following toxininduced astrocyte ablation in mice (Heithoff et al., 2021), which strongly suggests that astrocytic endfeet do indeed play a central role in maintaining the physical integrity of the BBB.
Together, the findings described above lead to the important question of whether or not disruptions in $\mathrm{Ca}^{2+}$ signals in astrocytic endfeet could compromise the established dependence of tight junction proteins (TJPs) on $\mathrm{Ca}^{2+}$ (Stuart et al., 1994; Brown and Davis, 2002), thereby altering BBB integrity. Although there is currently no clear evidence for a causative role of aberrant endfoot $\mathrm{Ca}^{2+}$ signals in altering TJP biology in PD, the use of new imaging modalities such as multiphoton microscopes in combination with astrocyte-specific transgenic mice (Srinivasan et al., 2016) and genetically encoded $\mathrm{Ca}^{2+}$ sensors in astrocytes should enable an understanding of the role of aberrant astrocytic endfoot $\mathrm{Ca}^{2+}$ signals in TJP and BBB function during PD.

\section{CONCLUSION}

In this perspective review, we discuss potential pathological mechanisms during PD in which aberrant astrocytic $\mathrm{Ca}^{2+}$ signals cause either neuronal dysfunction, microglial activation, or a loss of BBB integrity (Figure 1). Although we do not discuss what triggers abnormal $\mathrm{Ca}^{2+}$ signals in astrocytes during PD in the first place, molecules such as ApoE4 and $\alpha$-synuclein likely initiate abnormal $\mathrm{Ca}^{2+}$ signaling in astrocytes via multiple and distinct mechanisms. It is therefore reasonable to hypothesize that once they are initiated, abnormal astrocytic $\mathrm{Ca}^{2+}$ signals cause further abnormalities in ApoE4 or $\alpha$-synuclein, thereby setting up a vicious feedback loop between aberrant astrocytic $\mathrm{Ca}^{2+}$ signaling and ApoE4 or $\alpha$-synuclein pathology in PD.

An additional point to note is that neurons, microglia and the $\mathrm{BBB}$ are also capable of directly interacting with each other, which would result in a complex network of multi-tiered pathological interactions. Based on this view, we predict that the coming decades will unravel specific mechanisms by which aberrant astrocytic $\mathrm{Ca}^{2+}$ signals modulate multi-tiered interactions between these seemingly distinct CNS compartments, eventually leading to neurodegeneration.

\section{AUTHOR CONTRIBUTIONS}

RS conceived and wrote the manuscript. RS and EB compiled and edited the manuscript. EB created the figures with input from RS. Both authors contributed to the article and approved the submitted version.

\section{FUNDING}

This work was partially funded by the National Institutes of Health (NIH/NINDS R01 NS115809).

\section{ACKNOWLEDGMENTS}

We thank Taylor E. Huntington for providing the astrocyte image in Figure 1. Supported by a National Institutes of Health (NIH) research grant (R01 NS115809) to RS. 


\section{REFERENCES}

Adermark, L., Lagstrom, O., Loften, A., Licheri, V., Havenang, A., Loi, E. A., et al. (2021). Astrocytes modulate extracellular neurotransmitter levels and excitatory neurotransmission in dorsolateral striatum via dopamine D2 receptor signaling. Neuropsychopharmacology doi: 10.1038/s41386-021-01232-x

Agarwal, A., Wu, P. H., Hughes, E. G., Fukaya, M., Tischfield, M. A., Langseth, A. J., et al. (2017). Transient Opening of the Mitochondrial Permeability Transition Pore Induces Microdomain Calcium Transients in Astrocyte Processes. Neuron 93, 587-605.e7. doi: 10.1016/j.neuron.2016.12.034

Al-Bachari, S., Naish, J. H., Parker, G. J. M., Emsley, H. C. A., and Parkes, L. M. (2020). Blood-Brain Barrier Leakage Is Increased in Parkinson's Disease. Front. Physiol. 11:593026. doi: 10.3389/fphys.2020.593026

Ambrosi, G., Cerri, S., and Blandini, F. (2014). A further update on the role of excitotoxicity in the pathogenesis of Parkinson's disease. J. Neural Transm. 121, 849-859. doi: 10.1007/s00702-013-1149-z

Arizono, M., Inavalli, V., Panatier, A., Pfeiffer, T., Angibaud, J., Levet, F., et al. (2020). Structural basis of astrocytic $\mathrm{Ca}(2+)$ signals at tripartite synapses. Nat. Commun. 11:1906. doi: 10.1038/s41467-020-15648-4

Barcia, C., Sanchez Bahillo, A., Fernandez-Villalba, E., Bautista, V., Poza, Y. P. M., Fernandez-Barreiro, A., et al. (2004). Evidence of active microglia in substantia nigra pars compacta of parkinsonian monkeys 1 year after MPTP exposure. Glia 46, 402-409. doi: 10.1002/glia.20015

Barkholt, P., Sanchez-Guajardo, V., Kirik, D., and Romero-Ramos, M. (2012). Long-term polarization of microglia upon alpha-synuclein overexpression in nonhuman primates. Neuroscience 208, 85-96. doi: 10.1016/j.neuroscience. 2012.02.004

Bartels, A. L., Willemsen, A. T., Doorduin, J., De Vries, E. F., Dierckx, R. A., and Leenders, K. L. (2010). [11C]-PK11195 PET: quantification of neuroinflammation and a monitor of anti-inflammatory treatment in Parkinson's disease?. Parkinsonism Relat. Disord. 16, 57-59. doi: 10.1016/j. parkreldis.2009.05.005

Bartels, T., De Schepper, S., and Hong, S. (2020). Microglia modulate neurodegeneration in Alzheimer's and Parkinson's diseases. Science 370, 66-69. doi: $10.1126 /$ science.abb8587

Blaylock, R. L. (2017). Parkinson's disease: microglial/macrophage-induced immunoexcitotoxicity as a central mechanism of neurodegeneration. Surg. Neurol. Int. 8:65. doi: 10.4103/sni.sni_441_16

Bohlen, C. J., Friedman, B. A., Dejanovic, B., and Sheng, M. (2019). Microglia in Brain Development, Homeostasis, and Neurodegeneration. Annu. Rev. Genet. 53, 263-288. doi: 10.1146/annurev-genet-112618-043515

Booth, H. D. E., Hirst, W. D., and Wade-Martins, R. (2017). The Role of Astrocyte Dysfunction in Parkinson's Disease Pathogenesis. Trends Neurosci. 40, 358-370. doi: $10.1016 /$ j.tins.2017.04.001

Brown, R. C., and Davis, T. P. (2002). Calcium modulation of adherens and tight junction function: a potential mechanism for blood-brain barrier disruption after stroke. Stroke 33, 1706-1711. doi: 10.1161/01.STR.0000016405.06729.83

Bruijn, L. I., Becher, M. W., Lee, M. K., Anderson, K. L., Jenkins, N. A., Copeland, N. G., et al. (1997). ALS-linked SOD1 mutant G85R mediates damage to astrocytes and promotes rapidly progressive disease with SOD1-containing inclusions. Neuron 18, 327-338. doi: 10.1016/S0896-6273(00)80272-X

Bushong, E. A., Martone, M. E., Jones, Y. Z., and Ellisman, M. H. (2002). Protoplasmic astrocytes in CA1 stratum radiatum occupy separate anatomical domains. J. Neurosci. 22, 183-192. doi: 10.1523/JNEUROSCI.22-01-00183. 2002

Cardenas, C., Miller, R. A., Smith, I., Bui, T., Molgo, J., Muller, M., et al. (2010). Essential regulation of cell bioenergetics by constitutive InsP3 receptor Ca2+ transfer to mitochondria. Cell 142, 270-283. doi: 10.1016/j.cell.2010.06.007

Choi, I., Zhang, Y., Seegobin, S. P., Pruvost, M., Wang, Q., Purtell, K., et al. (2020). Microglia clear neuron-released alpha-synuclein via selective autophagy and prevent neurodegeneration. Nat. Commun. 11:1386. doi: 10.1038/s41467-02015119-w

Chotibut, T., Meadows, S., Kasanga, E. A., Mcinnis, T., Cantu, M. A., Bishop, C., et al. (2017). Ceftriaxone reduces L-dopa-induced dyskinesia severity in 6-hydroxydopamine parkinson's disease model. Mov. Disord. 32, 1547-1556. doi: $10.1002 / \mathrm{mds} .27077$

Corkrum, M., Covelo, A., Lines, J., Bellocchio, L., Pisansky, M., Loke, K., et al. (2020). Dopamine-Evoked Synaptic Regulation in the Nucleus Accumbens
Requires Astrocyte Activity. Neuron 105:e1035. doi: 10.1016/j.neuron.2019.12. 026

Covelo, A., and Araque, A. (2018). Neuronal activity determines distinct gliotransmitter release from a single astrocyte. Elife 7:e32237. doi: 10.7554/ eLife.32237.018

Czlonkowska, A., Kohutnicka, M., Kurkowska-Jastrzebska, I., and Czlonkowski, A. (1996). Microglial reaction in MPTP (1-methyl-4phenyl-1,2,3,6-tetrahydropyridine) induced Parkinson's disease mice model. Neurodegeneration 5, 137-143. doi: 10.1006/neur.1996.0020

Davis, A. A., Inman, C. E., Wargel, Z. M., Dube, U., Freeberg, B. M., Galluppi, A., et al. (2020). APOE genotype regulates pathology and disease progression in synucleinopathy. Sci. Transl. Med. 12:eaay3069. doi: 10.1126/scitranslmed. aay3069

Deemyad, T., Luthi, J., and Spruston, N. (2018). Astrocytes integrate and drive action potential firing in inhibitory subnetworks. Nat. Commun. 9:4336. doi: 10.1038/s41467-018-06338-3

Derouiche, A., Haseleu, J., and Korf, H. W. (2015). Fine Astrocyte Processes Contain Very Small Mitochondria: glial Oxidative Capability May Fuel Transmitter Metabolism. Neurochem. Res. 40, 2402-2413. doi: 10.1007/s11064015-1563-8

Eulenburg, V., and Gomeza, J. (2010). Neurotransmitter transporters expressed in glial cells as regulators of synapse function. Brain Res. Rev. 63, 103-112. doi: 10.1016/j.brainresrev.2010.01.003

Fan, Y., Kong, H., Shi, X., Sun, X., Ding, J., Wu, J., et al. (2008). Hypersensitivity of aquaporin 4-deficient mice to 1-methyl-4-phenyl-1,2,3,6-tetrahydropyrindine and astrocytic modulation. Neurobiol. Aging 29, 1226-1236. doi: 10.1016/j. neurobiolaging.2007.02.015

Freire, C., and Koifman, S. (2012). Pesticide exposure and Parkinson's disease: epidemiological evidence of association. Neurotoxicology 33, 947-971. doi: 10.1016/j.neuro.2012.05.011

Fujii, Y., Maekawa, S., and Morita, M. (2017). Astrocyte calcium waves propagate proximally by gap junction and distally by extracellular diffusion of ATP released from volume-regulated anion channels. Sci. Rep. 7:13115. doi: 10.1038/ s41598-017-13243-0

Gerhard, A., Pavese, N., Hotton, G., Turkheimer, F., Es, M., Hammers, A., et al. (2006). In vivo imaging of microglial activation with [11C](R)-PK11195 PET in idiopathic Parkinson's disease. Neurobiol. Dis. 21, 404-412. doi: 10.1016/j.nbd. 2005.08.002

Gray, M. T., and Woulfe, J. M. (2015). Striatal blood-brain barrier permeability in Parkinson's disease. J. Cereb. Blood Flow Metab. 35, 747-750. doi: 10.1038/ jcbfm.2015.32

Haustein, M. D., Kracun, S., Lu, X. H., Shih, T., Jackson-Weaver, O., Tong, X., et al. (2014). Conditions and constraints for astrocyte calcium signaling in the hippocampal mossy fiber pathway. Neuron 82, 413-429. doi: 10.1016/j.neuron. 2014.02.041

Heithoff, B. P., George, K. K., Phares, A. N., Zuidhoek, I. A., Munoz-Ballester, C., and Robel, S. (2021). Astrocytes are necessary for blood-brain barrier maintenance in the adult mouse brain. Glia 69, 436-472. doi: 10.1002/glia. 23908

Hoenen, C., Gustin, A., Birck, C., Kirchmeyer, M., Beaume, N., Felten, P., et al. (2016). Alpha-Synuclein Proteins Promote Pro-Inflammatory Cascades in Microglia: stronger Effects of the A53T Mutant. PLoS One 11:e0162717. doi: 10.1371/journal.pone.0162717

Hoshi, A., Tsunoda, A., Tada, M., Nishizawa, M., Ugawa, Y., and Kakita, A. (2017) Expression of Aquaporin 1 and Aquaporin 4 in the Temporal Neocortex of Patients with Parkinson's Disease. Brain Pathol. 27, 160-168. doi: 10.1111/bpa. 12369

Hubbard, J. A., Hsu, M. S., Seldin, M. M., and Binder, D. K. (2015). Expression of the Astrocyte Water Channel Aquaporin-4 in the Mouse Brain. ASN Neuro 7:1759091415605486. doi: 10.1177/1759091415605486

Huntington, T. E., and Srinivasan, R. (2021). Astrocytic mitochondria in adult mouse brain slices show spontaneous calcium influx events with unique properties. Cell Calcium 96:102383. doi: 10.1016/j.ceca.2021.10 2383

Ibanez, I., Bartolome-Martin, D., Piniella, D., Gimenez, C., and Zafra, F. (2019) Activity dependent internalization of the glutamate transporter GLT-1 requires calcium entry through the NCX sodium/calcium exchanger. Neurochem. Int. 123, 125-132. doi: 10.1016/j.neuint.2018.03.012 
Kanaan, N. M., Kordower, J. H., and Collier, T. J. (2008). Age and regionspecific responses of microglia, but not astrocytes, suggest a role in selective vulnerability of dopamine neurons after 1-methyl-4-phenyl-1,2,3,6tetrahydropyridine exposure in monkeys. Glia 56, 1199-1214. doi: 10.1002/ glia. 20690

Kitchen, P., Salman, M. M., Halsey, A. M., Clarke-Bland, C., Macdonald, J. A., Ishida, H., et al. (2020). Targeting Aquaporin-4 Subcellular Localization to Treat Central Nervous System Edema. Cell 181, 784-799.e19. doi: 10.1016/j.cell.2020. 03.037

Kockx, M., Guo, D. L., Huby, T., Lesnik, P., Kay, J., Sabaretnam, T., et al. (2007). Secretion of apolipoprotein $\mathrm{E}$ from macrophages occurs via a protein kinase A and calcium-dependent pathway along the microtubule network. Circ. Res. 101, 607-616. doi: 10.1161/CIRCRESAHA.107.157198

Lam, H. A., Wu, N., Cely, I., Kelly, R. L., Hean, S., Richter, F., et al. (2011). Elevated tonic extracellular dopamine concentration and altered dopamine modulation of synaptic activity precede dopamine loss in the striatum of mice overexpressing human alpha-synuclein. J. Neurosci. Res. 89, 1091-1102. doi: $10.1002 /$ jnr.22611

Lambert, J. C., Ibrahim-Verbaas, C. A., Harold, D., Naj, A. C., Sims, R., Bellenguez, C., et al. (2013). Meta-analysis of 74,046 individuals identifies 11 new susceptibility loci for Alzheimer's disease. Nat. Genet. 45, 1452-1458. doi: 10.1038/ng.2802

Lehre, K. P., and Danbolt, N. C. (1998). The number of glutamate transporter subtype molecules at glutamatergic synapses: chemical and stereological quantification in young adult rat brain. J. Neurosci. 18, 8751-8757. doi: 10.1523/ JNEUROSCI.18-21-08751.1998

Lewerenz, J., and Maher, P. (2015). Chronic Glutamate Toxicity in Neurodegenerative Diseases-What is the Evidence?. Front. Neurosci. 9:469. doi: 10.3389/fnins.2015.00469

Li, Q., and Barres, B. A. (2018). Microglia and macrophages in brain homeostasis and disease. Nat. Rev. Immunol. 18, 225-242. doi: 10.1038/nri.2017.125

Lindstrom, V., Gustafsson, G., Sanders, L. H., Howlett, E. H., Sigvardson, J., Kasrayan, A., et al. (2017). Extensive uptake of alpha-synuclein oligomers in astrocytes results in sustained intracellular deposits and mitochondrial damage. Mol. Cell Neurosci. 82, 143-156. doi: 10.1016/j.mcn.2017.04.009

Liu, C. C., Liu, C. C., Kanekiyo, T., Xu, H., and Bu, G. (2013). Apolipoprotein E and Alzheimer disease: risk, mechanisms and therapy. Nat. Rev. Neurol. 9, 106-118. doi: 10.1038/nrneurol.2012.263

Maezawa, I., Nivison, M., Montine, K. S., Maeda, N., and Montine, T. J. (2006). Neurotoxicity from innate immune response is greatest with targeted replacement of $\mathrm{E} 4$ allele of apolipoprotein $\mathrm{E}$ gene and is mediated by microglial p38MAPK. FASEB J. 20, 797-799. doi: 10.1096/fj.05-5423fje

McGeer, P. L., Itagaki, S., Boyes, B. E., and Mcgeer, E. G. (1988). Reactive microglia are positive for HLA-DR in the substantia nigra of Parkinson's and Alzheimer's disease brains. Neurology 38, 1285-1291. doi: 10.1212/WNL.38.8.1285

McGeer, P. L., Schwab, C., Parent, A., and Doudet, D. (2003). Presence of reactive microglia in monkey substantia nigra years after 1-methyl-4-phenyl-1,2,3,6tetrahydropyridine administration. Ann. Neurol. 54, 599-604. doi: 10.1002/ana. 10728

Moye, S. L., Diaz-Castro, B., Gangwani, M. R., and Khakh, B. S. (2019). Visualizing Astrocyte Morphology Using Lucifer Yellow Iontophoresis. J. Vis. Exp. 151:e60225. doi: 10.3791/60225

Oberheim, N. A., Takano, T., Han, X., He, W., Lin, J. H., Wang, F., et al. (2009). Uniquely hominid features of adult human astrocytes. J. Neurosci. 29, 3276-3287. doi: 10.1523/JNEUROSCI.4707-08.2009

Okubo, Y., Kanemaru, K., Suzuki, J., Kobayashi, K., Hirose, K., and Iino, M. (2019). Inositol 1,4,5-trisphosphate receptor type 2-independent $\mathrm{Ca}(2+)$ release from the endoplasmic reticulum in astrocytes. Glia 67, 113-124. doi: 10.1002/glia. 23531

Paillusson, S., Gomez-Suaga, P., Stoica, R., Little, D., Gissen, P., Devine, M. J., et al. (2017). alpha-Synuclein binds to the ER-mitochondria tethering protein VAPB to disrupt $\mathrm{Ca}(2+)$ homeostasis and mitochondrial ATP production. Acta Neuropathol. 134, 129-149. doi: 10.1007/s00401-017-1704-z

Pirttimaki, T. M., Sims, R. E., Saunders, G., Antonio, S. A., Codadu, N. K., and Parri, H. R. (2017). Astrocyte-Mediated Neuronal Synchronization Properties Revealed by False Gliotransmitter Release. J. Neurosci. 37, 9859-9870. doi: 10.1523/JNEUROSCI.2761-16.2017
Poewe, W., Seppi, K., Tanner, C. M., Halliday, G. M., Brundin, P., Volkmann, J., et al. (2017). Parkinson disease. Nat. Rev. Dis. Primers 3:17013. doi: 10.1038/ nrdp. 2017.13

Ramos-Gonzalez, P., Mato, S., Chara, J. C., Verkhratsky, A., Matute, C., and Cavaliere, F. (2021). Astrocytic atrophy as a pathological feature of Parkinson's disease with LRRK2 mutation. NPJ Parkinsons Dis. 7:31. doi: 10.1038/s41531021-00175-w

Rossi, D., Brambilla, L., Valori, C. F., Roncoroni, C., Crugnola, A., Yokota, T., et al. (2008). Focal degeneration of astrocytes in amyotrophic lateral sclerosis. Cell Death Differ. 15, 1691-1700. doi: 10.1038/cdd.2008.99

Salman, M. M., Kitchen, P., Woodroofe, M. N., Brown, J. E., Bill, R. M., Conner, A. C., et al. (2017). Hypothermia increases aquaporin 4 (AQP4) plasma membrane abundance in human primary cortical astrocytes via a calcium/transient receptor potential vanilloid 4 (TRPV4)- and calmodulinmediated mechanism. Eur. J. Neurosci. 46, 2542-2547. doi: 10.1111/ejn.13723

Sanchez-Guajardo, V., Febbraro, F., Kirik, D., and Romero-Ramos, M. (2010). Microglia acquire distinct activation profiles depending on the degree of alphasynuclein neuropathology in a rAAV based model of Parkinson's disease. PLoS One 5:e8784. doi: 10.1371/journal.pone.0008784

Sasaki, T., Ishikawa, T., Abe, R., Nakayama, R., Asada, A., Matsuki, N., et al. (2014). Astrocyte calcium signalling orchestrates neuronal synchronization in organotypic hippocampal slices. J. Physiol. 592, 2771-2783. doi: 10.1113/ jphysiol.2014.272864

Schapira, A. H. V., Chaudhuri, K. R., and Jenner, P. (2017). Non-motor features of Parkinson disease. Nat. Rev. Neurosci. 18, 435-450. doi: 10.1038/nrn.2017.62

Schubert, D., and Piasecki, D. (2001). Oxidative glutamate toxicity can be a component of the excitotoxicity cascade. J. Neurosci. 21, 7455-7462. doi: 10. 1523/JNEUROSCI.21-19-07455.2001

Semyanov, A., Henneberger, C., and Agarwal, A. (2020). Making sense of astrocytic calcium signals - from acquisition to interpretation. Nat. Rev. Neurosci. 21, 551-564. doi: 10.1038/s41583-020-0361-8

Semyanov, A., and Verkhratsky, A. (2021). Astrocytic processes: from tripartite synapses to the active milieu. Trends Neurosci. 44, 781-792. doi: 10.1016/j.tins. 2021.07.006

Sharma, A., Kazim, S. F., Larson, C. S., Ramakrishnan, A., Gray, J. D., Mcewen, B. S., et al. (2019). Divergent roles of astrocytic versus neuronal EAAT2 deficiency on cognition and overlap with aging and Alzheimer's molecular signatures. Proc. Natl. Acad. Sci. U. S. A. 116, 21800-21811. doi: 10.1073/pnas. 1903566116

Shigetomi, E., Jackson-Weaver, O., Huckstepp, R. T., O'dell, T. J., and Khakh, B. S. (2013). TRPA1 channels are regulators of astrocyte basal calcium levels and long-term potentiation via constitutive D-serine release. J. Neurosci. 33, 10143-10153. doi: 10.1523/JNEUROSCI.5779-12.2013

Shigetomi, E., Saito, K., Sano, F., and Koizumi, S. (2019). Aberrant Calcium Signals in Reactive Astrocytes: a Key Process in Neurological Disorders. Int. J. Mol. Sci. 20:996. doi: 10.3390/ijms20040996

Shigetomi, E., Tong, X., Kwan, K. Y., Corey, D. P., and Khakh, B. S. (2011). TRPA1 channels regulate astrocyte resting calcium and inhibitory synapse efficacy through GAT-3. Nat. Neurosci. 15, 70-80. doi: 10.1038/nn.3000

Smith, A. J., and Verkman, A. S. (2015). Superresolution Imaging of Aquaporin4 Cluster Size in Antibody-Stained Paraffin Brain Sections. Biophys. J. 109, 2511-2522. doi: 10.1016/j.bpj.2015.10.047

Srinivasan, R., Huang, B. S., Venugopal, S., Johnston, A. D., Chai, H., Zeng, H., et al. (2015). $\mathrm{Ca}(2+)$ signaling in astrocytes from Ip3r2(-/-) mice in brain slices and during startle responses in vivo. Nat. Neurosci. 18, 708-717. doi: 10.1038/ nn. 4001

Srinivasan, R., Lu, T. Y., Chai, H., Xu, J., Huang, B. S., Golshani, P., et al. (2016). New Transgenic Mouse Lines for Selectively Targeting Astrocytes and Studying Calcium Signals in Astrocyte Processes In Situ and In Vivo. Neuron 92, 1181-1195. doi: 10.1016/j.neuron.2016.11.030

Stokholm, M. G., Iranzo, A., Ostergaard, K., Serradell, M., Otto, M., Svendsen, K. B., et al. (2017). Assessment of neuroinflammation in patients with idiopathic rapid-eye-movement sleep behaviour disorder: a case-control study. Lancet Neurol. 16, 789-796. doi: 10.1016/S1474-4422(17)30173-4

Stuart, R. O., Sun, A., Panichas, M., Hebert, S. C., Brenner, B. M., and Nigam, S. K. (1994). Critical role for intracellular calcium in tight junction biogenesis. J. Cell Physiol. 159, 423-433. doi: 10.1002/jcp.1041590306 
Sun, H., Liang, R., Yang, B., Zhou, Y., Liu, M., Fang, F., et al. (2016). Aquaporin4 mediates communication between astrocyte and microglia: implications of neuroinflammation in experimental Parkinson's disease. Neuroscience 317, 6575. doi: 10.1016/j.neuroscience.2016.01.003

Sun, Y., Wu, S., Bu, G., Onifade, M. K., Patel, S. N., Ladu, M. J., et al. (1998). Glial fibrillary acidic protein-apolipoprotein E (apoE) transgenic mice: astrocytespecific expression and differing biological effects of astrocyte-secreted apoE3 and apoE4 lipoproteins. J. Neurosci. 18, 3261-3272. doi: 10.1523/JNEUROSCI. 18-09-03261.1998

Tham, D. K., Joshi, B., and Moukhles, H. (2016). Aquaporin-4 Cell-Surface Expression and Turnover Are Regulated by Dystroglycan, Dynamin, and the Extracellular Matrix in Astrocytes. PLoS One 11:e0165439. doi: 10.1371/ journal.pone.0165439

Tong, X., Ao, Y., Faas, G. C., Nwaobi, S. E., Xu, J., Haustein, M. D., et al. (2014). Astrocyte Kir4.1 ion channel deficits contribute to neuronal dysfunction in Huntington's disease model mice. Nat. Neurosci. 17, 694-703. doi: 10.1038/nn. 3691

Valori, C. F., Brambilla, L., Martorana, F., and Rossi, D. (2014). The multifaceted role of glial cells in amyotrophic lateral sclerosis. Cell Mol. Life Sci. 71, 287-297. doi: 10.1007/s00018-013-1429-7

van Hall, G., Stromstad, M., Rasmussen, P., Jans, O., Zaar, M., Gam, C., et al. (2009). Blood lactate is an important energy source for the human brain. J. Cereb. Blood Flow Metab. 29, 1121-1129. doi: 10.1038/jcbfm.2009.35

Verkhratsky, A., and Nedergaard, M. (2018). Physiology of Astroglia. Physiol. Rev. 98, 239-389. doi: 10.1152/physrev.00042.2016

Verkhratsky, A., Semyanov, A., and Zorec, R. (2020). Physiology of Astroglial Excitability. Function 1:zqaa016. doi: 10.1093/function/zqaa016

Verkhratsky, A., Zorec, R., Rodriguez, J. J., and Parpura, V. (2016). Astroglia dynamics in ageing and Alzheimer's disease. Curr. Opin. Pharmacol. 26, 74-79. doi: 10.1016/j.coph.2015.09.011

Verkhratsky, A., Zorec, R., Rodriguez, J. J., and Parpura, V. (2017). Neuroglia: functional Paralysis and Reactivity in Alzheimer's Disease and Other Neurodegenerative Pathologies. Adv. Neurobiol. 15, 427-449. doi: 10.1007/9783-319-57193-5_17

Vitek, M. P., Brown, C. M., and Colton, C. A. (2009). APOE genotype-specific differences in the innate immune response. Neurobiol. Aging 30, 1350-1360. doi: 10.1016/j.neurobiolaging.2007.11.014

von Bartheld, C. S., Bahney, J., and Herculano-Houzel, S. (2016). The search for true numbers of neurons and glial cells in the human brain: a review of 150 years of cell counting. J. Comp. Neurol. 524, 3865-3895. doi: 10.1002/cne.24040

Wang, J., Wang, F., Mai, D., and Qu, S. (2020). Molecular Mechanisms of Glutamate Toxicity in Parkinson's Disease. Front. Neurosci. 14:585584. doi: 10.3389/fnins.2020.585584

Wu, D. C., Jackson-Lewis, V., Vila, M., Tieu, K., Teismann, P., Vadseth, C., et al. (2002). Blockade of microglial activation is neuroprotective in the 1-methyl-4-phenyl-1,2,3,6-tetrahydropyridine mouse model of Parkinson disease. J. Neurosci. 22, 1763-1771. doi: 10.1523/JNEUROSCI.22-05-01763. 2002

Xin, W., Schuebel, K. E., Jair, K. W., Cimbro, R., De Biase, L. M., Goldman, D., et al. (2019). Ventral midbrain astrocytes display unique physiological features and sensitivity to dopamine D2 receptor signaling. Neuropsychopharmacology 44, 344-355. doi: 10.1038/s41386-018-0151-4

Xu, Q., Bernardo, A., Walker, D., Kanegawa, T., Mahley, R. W., and Huang, Y. (2006). Profile and regulation of apolipoprotein E (ApoE) expression in the CNS in mice with targeting of green fluorescent protein gene to the ApoE locus. J. Neurosci. 26, 4985-4994. doi: 10.1523/JNEUROSCI.5476-05. 2006

Yamazaki, Y., Zhao, N., Caulfield, T. R., Liu, C. C., and Bu, G. (2019). Apolipoprotein $\mathrm{E}$ and Alzheimer disease: pathobiology and targeting strategies. Nat. Rev. Neurol. 15, 501-518. doi: 10.1038/s41582-019-0228-7

Zhang, J., Yang, B., Sun, H., Zhou, Y., Liu, M., Ding, J., et al. (2016). Aquaporin-4 deficiency diminishes the differential degeneration of midbrain dopaminergic neurons in experimental Parkinson's disease. Neurosci. Lett. 614, 7-15. doi: 10.1016/j.neulet.2015.12.057

Zhang, Y., Meng, X., Jiao, Z., Liu, Y., Zhang, X., and Qu, S. (2020). Generation of a Novel Mouse Model of Parkinson's Disease via Targeted Knockdown of Glutamate Transporter GLT-1 in the Substantia Nigra. ACS Chem. Neurosci. 11, 406-417. doi: 10.1021/acschemneuro.9b00609

Zhao, N., Attrebi, O. N., Ren, Y., Qiao, W., Sonustun, B., Martens, Y. A., et al. (2020). APOE4 exacerbates alpha-synuclein pathology and related toxicity independent of amyloid. Sci. Transl. Med. 12:eaay1809. doi: 10.1126/ scitranslmed.aay1809

Zhou, B., Zuo, Y. X., and Jiang, R. T. (2019). Astrocyte morphology: diversity, plasticity, and role in neurological diseases. CNS Neurosci. Ther. 25, 665-673. doi: $10.1111 / \mathrm{cns} .13123$

Conflict of Interest: The authors declare that the research was conducted in the absence of any commercial or financial relationships that could be construed as a potential conflict of interest.

Publisher's Note: All claims expressed in this article are solely those of the authors and do not necessarily represent those of their affiliated organizations, or those of the publisher, the editors and the reviewers. Any product that may be evaluated in this article, or claim that may be made by its manufacturer, is not guaranteed or endorsed by the publisher.

Copyright (c) 2022 Bancroft and Srinivasan. This is an open-access article distributed under the terms of the Creative Commons Attribution License (CC BY). The use, distribution or reproduction in other forums is permitted, provided the original author(s) and the copyright owner(s) are credited and that the original publication in this journal is cited, in accordance with accepted academic practice. No use, distribution or reproduction is permitted which does not comply with these terms. 